The Astrophysical Journal, 689:148-152, 2008 December 10

(C) 2008. The American Astronomical Society. All rights reserved. Printed in U.S.A.

\title{
DETECTION OF DENSE MOLECULAR GAS IN INTERARM SPURS IN M51
}

\author{
Stuartt Corder, ${ }^{1}$ Kartik Sheth, ${ }^{1,2}$ Nicholas Z. Scoville, ${ }^{1}$ Jin Koda, $^{1}$ Stuart N. Vogel, ${ }^{3}$ and Eve Ostriker ${ }^{3}$ \\ Received 2008 May 29; accepted 2008 July 17
}

\begin{abstract}
Spiral arm spurs are prominent features that have been observed in extinction and $8 \mu \mathrm{m}$ emission in nearby galaxies. In order to understand their molecular gas properties, we used the Owens Valley Radio Observatory (OVRO) to map the $\mathrm{CO}(1-0)$ emission in three spurs emanating from the inner northwestern spiral arm of M51. We report CO detections from all three spurs. The molecular gas mass and surface density are $M_{\mathrm{H}_{2}} \sim 3 \times 10^{6} M_{\odot}$ and $\Sigma_{\mathrm{H}_{2}} \sim$ $50 M_{\odot} \mathrm{pc}^{-2}$. Thus, relative to the spiral arms, the spurs are extremely weak features. However, since the spurs are extended perpendicular to the spiral arms for $\sim 500 \mathrm{pc}$ and contain adequate fuel for star formation, they may be the birthplace for observed interarm $\mathrm{H}$ II regions. This reduces the requirement for the significant time delay that would be otherwise needed if the interarm star formation was initiated in the spiral arms. Larger maps of galaxies at similar depth are required to further understand the formation and evolution of these spurs and their role in star formationsuch data should be forthcoming with the new Combined Array for Research in Millimeter Astronomy (CARMA) and future Atacama Large Millimeter/submillimeter Array (ALMA) telescopes, and can be compared to several recent numerical simulations that have been examining the evolution of spiral arm spurs.
\end{abstract}

Subject headings: galaxies: individual (M51, NGC 5194) — galaxies: ISM — galaxies: spiral — ISM: structure — stars: formation

\section{INTRODUCTION}

One of the most striking features of the M51 mosaics from observations with the Hubble Space Telescope's (HST) Wide Field Planetary Camera and Advanced Camera for Surveys (ACS) is the presence of regularly spaced, dark spurs extending perpendicularly outward from the spiral arms (Scoville et al. 2001). With dust emission maps from the Spitzer Space Telescope, the spurs are also seen most prominently in the Infrared Array Camera (IRAC) $8 \mu \mathrm{m}$ maps of nearby galaxies (e.g., Fig. 1 of Brunner et al. 2008). In addition, the IRAC images also reveal a complex web of emission features which crisscross the spiral arms and the interarm regions. The $8 \mu \mathrm{m}$ data primarily trace polycyclic aromatic hydrocarbon $(\mathrm{PAH})$ emission that is believed to originate from surfaces of photodissociation regions (PDRs). The exact nature of these features is not well known, but these features have been noted in previous observational studies both in the Milky Way and in nearby galaxies. For instance, our own solar system is believed to lie in a spur that is an offshoot of the Sagittarius spiral arm (Weaver 1970). In a study of seven nearby spirals, including M51, Elmegreen (1980) measured an average pitch angle of $\sim 60^{\circ}$ and a width of $\sim 560 \mathrm{pc}$ for spurs, and concluded that they were likely to be long-lived features. These results were confirmed in the higher resolution, larger sample of La Vigne et al. (2006). Elmegreen (1979) also noted that spurs are often accompanied by a parallel string of $\mathrm{H}$ II regions and may trigger star formation. In barred spirals Sheth et al. (2000, 2002) found a correlation between spurs, which are normally seen upstream of bar dust lanes and $\mathrm{H}$ II regions on the leading (downstream) side of the dust lanes. They have argued that spurs are conducive to star formation because of their increased gas surface density relative to the surroundings and lower shear relative

\footnotetext{
${ }^{1}$ Department of Astronomy, California Institute of Technology, MS 105-24 Caltech, Pasadena, CA 91125.

${ }^{2}$ Spitzer Science Center and Department of Astronomy, California Institute of Technology, MS 220-6 Caltech, Pasadena, CA 91125.

3 Department of Astronomy, University of Maryland, College Park, MD $20742-2421$
}

to the bar dust lane. Note, however, that the bar dust lane spurs are usually on the trailing side of the dust lane, whereas the spiral arm spurs are on the leading side, which suggests that they may be a different phenomenon and have a different origin than the spiral arm spurs. In the most recent survey of nearby spirals, M. A. La Vigne et al. (2009, in preparation) find that spurs and feathers are common in disks with well-defined dust lanes and the spur separation increases with galactocentric radius. They find spurs in $20 \%$ of all nearby disks. Recent numerical models have shown that spurs form downstream from spiral arms under a variety of conditions (Kim \& Ostriker 2002, 2006; Wada \& Koda 2004; Dobbs \& Bonnell 2006; Shetty \& Ostriker 2006; Wada 2008).

In this paper we present observations of the $\mathrm{CO}(1-0)$ line emission in the inner, northwestern spiral arm and spurs, and measure the masses and average gas surface densities of the spurs. The millimeter data are the deepest observations of a field in M51 with the Owens Valley Radio Observatory's (OVRO) millimeter array. These data and analysis provide the groundwork for the expected large surveys with the Combined Array for Research in Millimeter Astronomy (CARMA) and the Atacama Large Millimeter/submillimeter Array (ALMA), which allow one to study galactic substructure over entire galaxy disks in nearby galaxies.

\section{OBSERVATIONS AND DATA REDUCTIONS}

Single-pointing, OVRO ${ }^{12} \mathrm{CO}(1-0, \lambda 2.6 \mathrm{~mm})$ observations were carried out between 2001 January and 2003 April, in the compact, low- and high-resolution configurations. These configurations resulted in projected baselines ranging from 4 to $92 \mathrm{k} \lambda$. The digital spectral correlator was configured for a resolution of $2.6 \mathrm{~km} \mathrm{~s}^{-1}$ with a total bandwidth of $332 \mathrm{~km} \mathrm{~s}^{-1}$. The quasar $1156+295$ was observed every 15-20 minutes for phase and amplitude gain calibration. The total on-source integration time was $54.5 \mathrm{hr}$. We reduced the data using the MMA software package (Scoville et al. 1993), and imaged the data using MIRIAD (Sault et al. 1995). The synthesized beam using robust weighting 
TABLE 1

M51 Properties

\begin{tabular}{|c|c|}
\hline Parameter & Value \\
\hline Center position (J2000.0) ................. & $\alpha=13^{\mathrm{h}} 29^{\mathrm{m}} 50.38^{\mathrm{s}}, \delta=47^{\circ} 12^{\prime} 02.28^{\prime \prime}$ \\
\hline 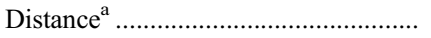 & $8.4 \mathrm{Mpc}$ \\
\hline Position angle.................................. & $170^{\circ}$ \\
\hline 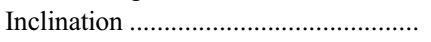 & $20^{\circ}$ \\
\hline 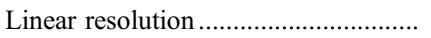 & $112 \times 85 \mathrm{pc}$ \\
\hline 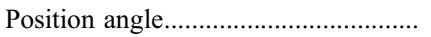 & $-57.9^{\circ}$ \\
\hline 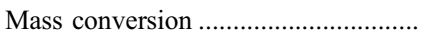 & $M=1.5 \times 10^{4} S \Delta v D^{2} M_{\odot}$ \\
\hline
\end{tabular}

a The value here is taken from Regan et al. (2001), but other values are reported in the literature which indicate an uncertainty of $25 \%$.

(Briggs 1995) is $2.76^{\prime \prime} \times 2.10^{\prime \prime}$. The FWHM of the primary beam is $1^{\prime}$. The spectral data were binned into $5 \mathrm{~km} \mathrm{~s}^{-1}$ channels for our analysis. The rms noise in a $5 \mathrm{~km} \mathrm{~s}^{-1}$ channel was $13 \mathrm{mJy} \mathrm{beam}^{-1}$. Table 1 lists parameters for M51 parameters used in this paper and the pointing center of the single OVRO field.

\section{RESULTS}

The velocity-integrated molecular gas emission is shown overlaid on a $V$-band $H S T$ image in Figure 1 for the field targeted by our OVRO observations. The optical image shows four spurs labeled spur 1, 2a, 2b, and 3. Molecular gas emission is associated with three (spur 1, 2a, and 3) of these four spurs. Two of the three spurs have very similar morphology, with a dense tip and an extended body which connects back to the base at the spur/ arm interface. Additional features in the interarm region are not labeled. The $\mathrm{CO}$ emission in the spiral arm is extremely bright with a steep gradient on the upstream and downstream edges. Peak emission in the arm is an order of magnitude brighter than in the spurs. The brightest spiral arm emission is in a ridge on the downstream side of the arm with distinct peaks of gas surface density that are labeled as arm peak 1,2 , and 3 . The channel maps for the $\mathrm{CO}$ emission are shown in Figure 2, where each of the spurs can be uniquely identified in a distinct set of channels (spur 1 from 380 to $440 \mathrm{~km} \mathrm{~s}^{-1}$, spur $2 \mathrm{a}$ from 430 to $455 \mathrm{~km} \mathrm{~s}^{-1}$, and spur 3 from 450 to $465 \mathrm{~km} \mathrm{~s}^{-1}$ ). Spur 1 has the most distinctive

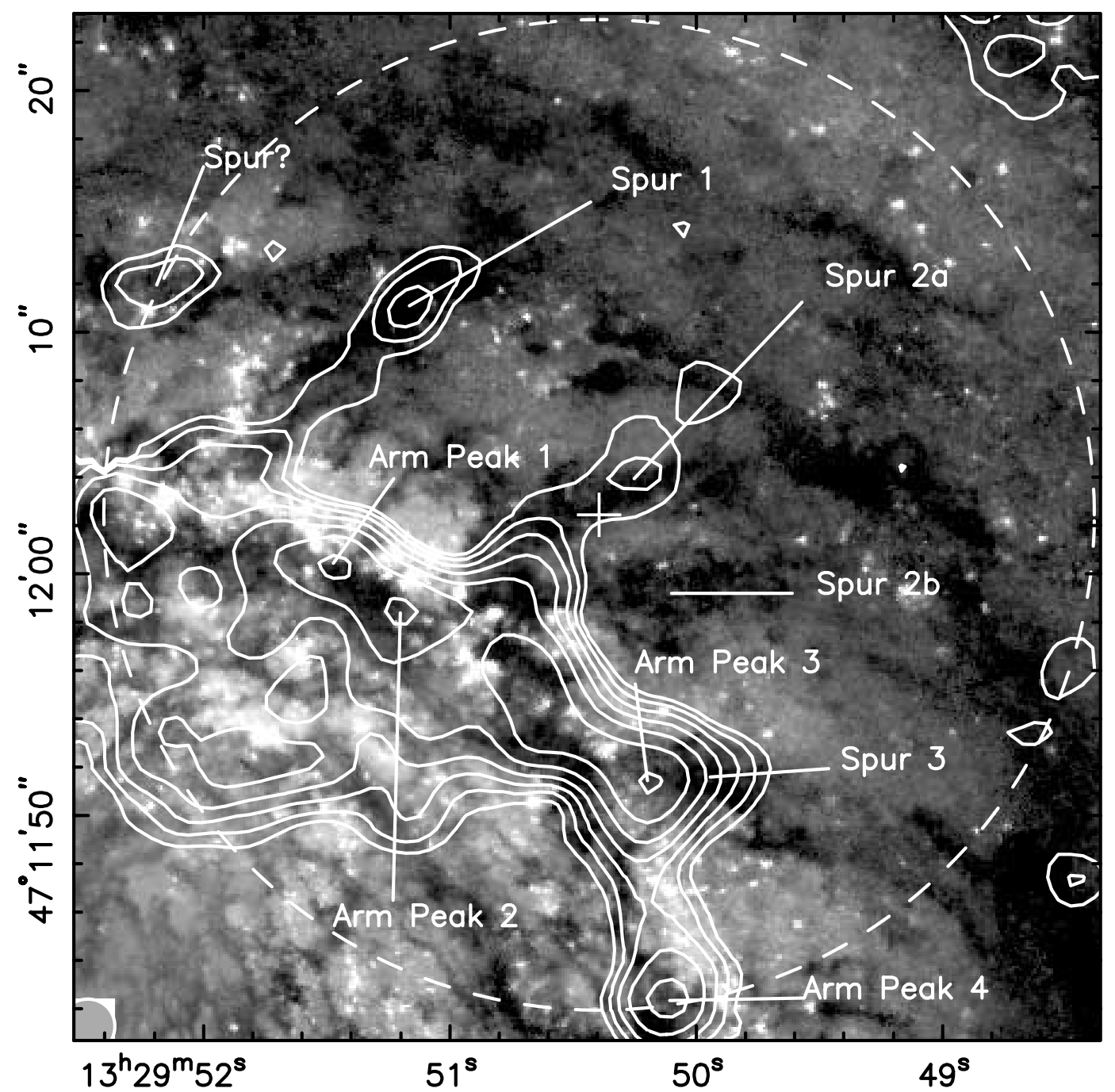

FIG. 1.-The $V$-band HST image of Scoville et al. (2001) with the velocity-integrated CO map from OVRO overlaid in contours. The contours are logarithmically spaced at $1 \mathrm{Jy} \mathrm{beam}^{-1} \mathrm{~km} \mathrm{~s}^{-1} \times 1.5^{n}$, where $n=0,1,2,3, \ldots 6$. The phase and pointing center is marked with a plus sign, and the dashed contour is the $70 \%$ gain of the OVRO primary beam. The three spurs in the OVRO field are labeled as spurs 1, 2a, and 3, and the three arm peaks used to determine density contrasts of spiral arm peaks

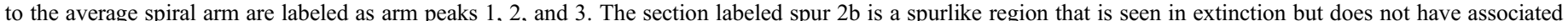
$\mathrm{CO}$ emission; the base of the spur might be arm peak 2 . Two other features, an arm peak 4 and a spur to the east of spur 1 , are also visible but are near the edge of the primary beam, and we do not include them in this study. 

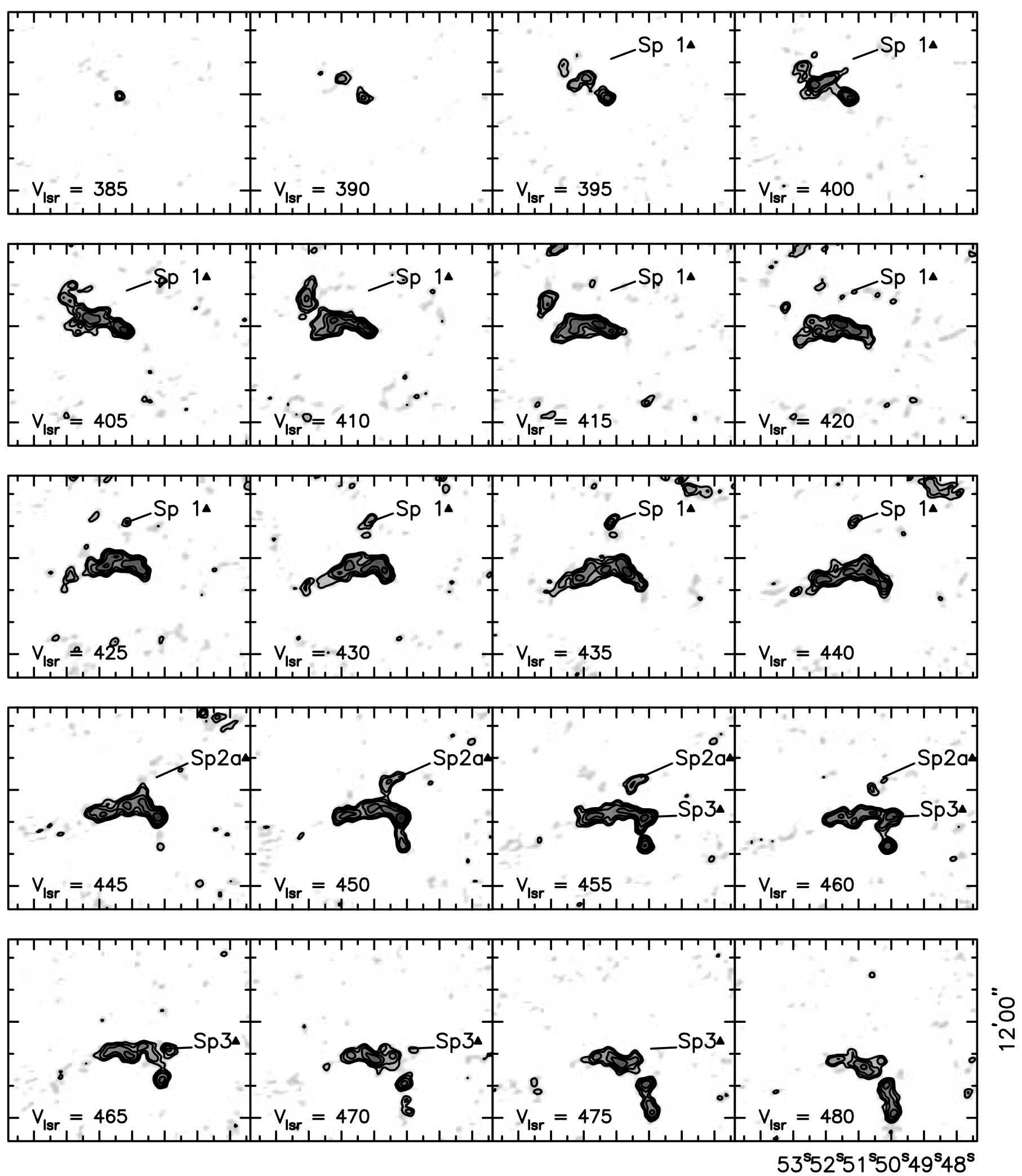

FIG. 2.-Channel maps for spurs from the OVRO data. The channels are $5 \mathrm{~km} \mathrm{~s}^{-1}$ wide. Spurs 1, 2a, and 3 are labeled as Sp1, Sp2a, and Sp3, respectively. Gray-scale emission starts at $2 \sigma$. The contours are at $1.5^{n} \times 15 \mathrm{mJy} \mathrm{beam}^{-1}$, with $n=3,4,5, \ldots$. The emission peak at the tip of spur 1 is at $11 \sigma$, while the base of this spur is seen at $\sim 5 \sigma$. Spur 2 a is detected at $9.5 \sigma$, and spur 3 has emission between 4 and $10 \sigma$, although separating spur from arm emission is difficult here. The peak of each spur is about 2-3.5 times lower than the peak emission in the arm in the same channel. 
TABLE 2

Spur Properties: Molecular Gas

\begin{tabular}{|c|c|c|c|c|c|}
\hline Spur & $\begin{array}{c}-\Delta \alpha^{\mathrm{a}} \\
\text { (s) }\end{array}$ & $\begin{array}{c}\Delta \delta^{\mathrm{a}} \\
(\operatorname{arcsec})\end{array}$ & $\begin{array}{c}\text { CO Flux } \\
\left(\mathrm{Jy} \mathrm{km} \mathrm{s}^{-1}\right)\end{array}$ & $\begin{array}{c}\text { Mass } \\
\left(10^{6} M_{\odot}\right)\end{array}$ & $\begin{array}{c}\Sigma \\
\left(M_{\odot} \mathrm{pc}^{-2}\right)\end{array}$ \\
\hline $1^{\mathrm{b}}$ & 1.32 & 28.16 & 1.69 & 1.8 & 71 \\
\hline 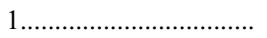 & $1.55-1.32$ & $24.66-28.16$ & 3.12 & 3.4 & 49 \\
\hline 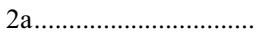 & $2.53-2.14$ & $16.24-20.69$ & 4.86 & 5.3 & 56 \\
\hline ..................... & $\sim 2.89$ & $\sim 8.75$ & 3.53 & $\simeq 3.9$ & $\simeq 72$ \\
\hline
\end{tabular}

a The first value is that of the spur base, while the second value is the spur tip.

b Values based wholly on the properties of the tip of the spur.

morphology and velocity structure. The tip of spur 1 , seen best in the channel maps from 415 to $430 \mathrm{~km} \mathrm{~s}^{-1}$, is $350 \mathrm{pc}$ away from the spiral arm and appears to be a coherent and dense region of radius $90 \mathrm{pc}^{2}$. Integrating over this area and velocity range gives a total CO flux of $1.69 \mathrm{Jy} \mathrm{km} \mathrm{s}^{-1}$, or a total gas mass of $\sim 1.8 \times 10^{6} M_{\odot}$, and a gas surface density of $71 M_{\odot} \mathrm{pc}^{-2}$.

Spur $2 \mathrm{a}$ and spur 3 have similar extensions in the channel maps as spur 1, although their tips are not as distant or distinctive from the base of each spurs. We detect $\mathrm{CO}$ emission over a length of 475,420 , and 155 pc along spurs $1,2 \mathrm{a}$, and 3 , re- spectively. Integrating over these regions we measure total spur masses of $3.4,5.3$, and $\sim 3.9 \times 10^{6} M_{\odot}$, respectively. The total area over which there is $\mathrm{CO}$ emission in the spurs ranges from 5.4 to $9.5 \times 10^{4} \mathrm{pc}^{2}$. Therefore the gas surface densities are 49 , 56 , and $>72 M_{\odot} \mathrm{pc}^{-2}$ for spurs $1,2 \mathrm{a}$, and 3 , respectively. The molecular gas properties of the spurs are summarized in Table 2. We do not detect CO emission from the spur labeled $2 b$. Since the spurs are identified in extinction and the geometry of the dust and stars is not known, the column density in this spur is likely to be low. It is plausible that the spiral arm peak 2 and the slight

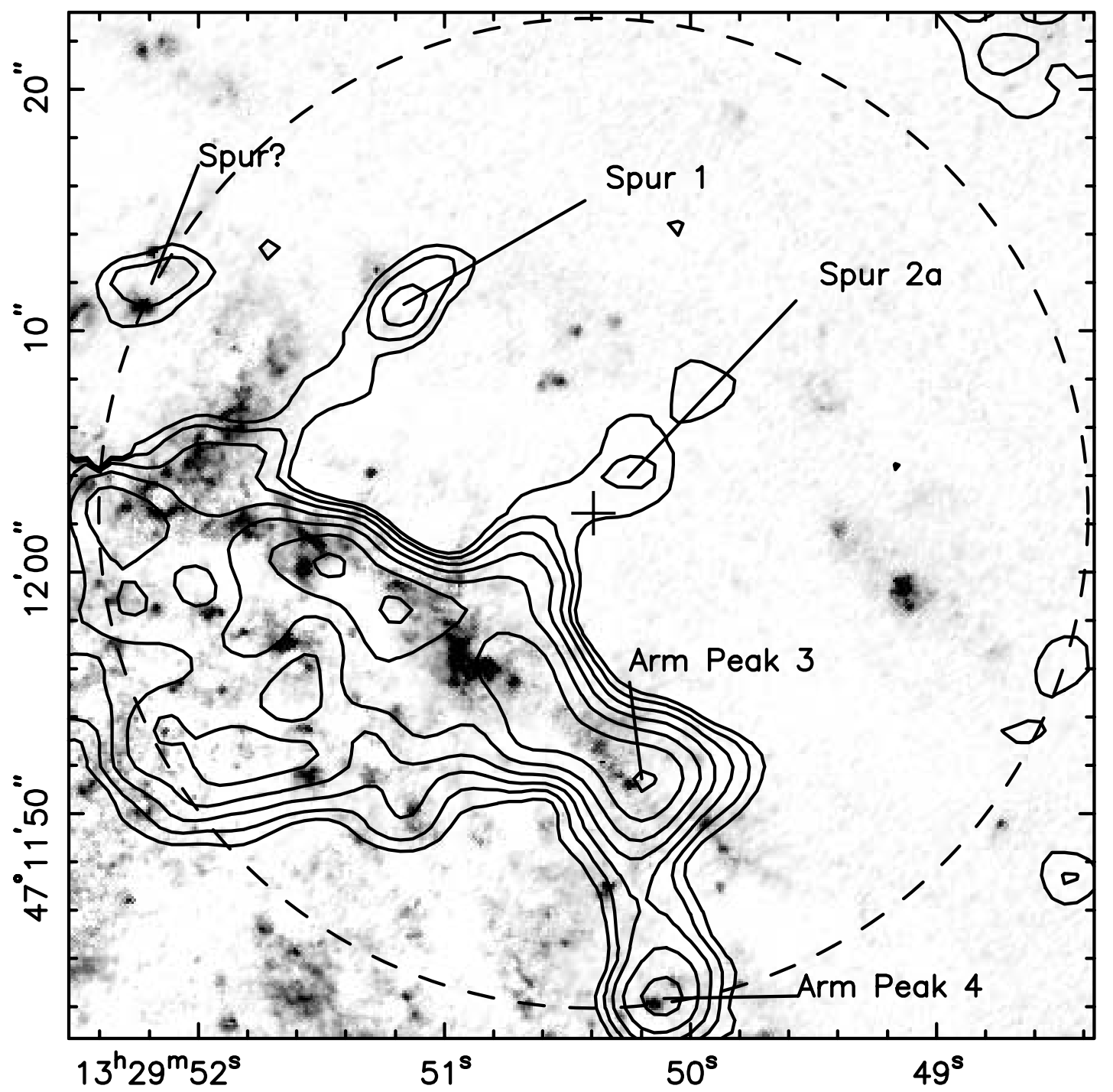

FIG. 3.-The H $\alpha$ HST image of Scoville et al. (2001) with CO emission contour map from OVRO overlaid. While slightly offset, the H $\alpha$ emission northeast of spur 1 is morphologically identical to the $\mathrm{CO}$ emission and is thought to be associated with the spur. 
extension to the northeast may be the base of spur $2 b$. Given the lack of $\mathrm{CO}$ detection, we put an upper limit of $6.0 \times 10^{5} M_{\odot}$ and $6 M_{\odot} \mathrm{pc}^{-2}$ on this spur.

\section{DISCUSSION}

The detection of CO emission in the spurs of M5I shows that the spurs are massive and dense structures with significant amounts of molecular gas. The most massive fragment in a spur in our data is at least as large as some of the largest giant molecular clouds/ associations in the Milky Way (Scoville \& Solomon 1975; Sanders et al. 1985; Dame et al. 1986; Sheth et al. 2008). The mean density in the arm, defined by the arm segment between spurs 1 and $2 \mathrm{a}$ extending to the edge of the emission to the southeast, is $300 M_{\odot} \mathrm{pc}^{-2}$. The peak density in the arm is a factor of 2 or more than this density, implying that, overall, the spurs are significantly weaker relative to the strong spiral arm emission. Since the spiral arm emission is so bright in M51, the spurs in M51 are likely to be brighter and more massive relative to spurs in other galaxies. Ongoing larger surveys with CARMA may shed light on the overall properties of spurs (e.g., J. Koda et al. 2009, in preparation; M. A. La Vigne et al. 2009, in preparation). Future higher resolution observations will help delineate the spur structures and provide detailed kinematics which are needed for distinguishing between the current models of spur formation and evolution.

The detection of molecular gas in spurs is significant for shedding light on the long-standing problem of the interarm $\mathrm{H}$ II regions. The widely accepted picture of star formation in spiral arms has been that clouds converge in the spiral arms, collide, and form giant molecular associations in which star formation is triggered. Both the newly formed stars and clouds then travel outward into the interarm region. There is an observed offset between the $\mathrm{H}$ II regions near the spiral arm and the peak of the molecular gas density in the arm. This same offset has been observed in other galaxies. Vogel et al. (1988) offered an explanation for the offset in terms of a time delay between the agglomeration of clouds in the spiral arm and the star formation activity, which is downstream from the spiral arms. However, this delay becomes increasingly unpalatable for interarm $\mathrm{H}$ II regions which are offset more than a few hundred parsecs from the spiral arm.
The detection of dense molecular gas in spurs may be the solution, since the spurs extend hundreds of parsecs into the interarm area. When we examine the structure at the tip of spur 1 we find that it is kinematically distinct from the base and extended body of the spur. The total mass in this structure is $1.8 \times 10^{6} M_{\odot}$. The structure itself has a diameter of $\sim 180 \mathrm{pc}$ and a line width of $10.4 \mathrm{~km} \mathrm{~s}^{-1}$. Its virial mass, about $4 \times 10^{6} M_{\odot}$, is greater than the mass measured from the $\mathrm{CO}$ flux. This suggests that the fragment may not be bound. If bound, it suggests that the tip has fragmented from the body of the spur and may be traveling independently in the interarm region. If unbound, the apparent spur tip may just be a congregation of lower mass clouds which may eventually diverge, then becoming undetectable at our image depth. Regardless of the nature of this spur tip, there is star formation associated with the tip as seen by the presence of $\mathrm{H} \alpha$ emission (see Fig. 3), implying that it can be the source of interarm $\mathrm{H}$ II regions. This explanation is also proposed in the modeling studies by Kim \& Ostriker $(2002,2006)$, in which they form clouds of masses $\sim 7 \times 10^{6} M_{\odot}$ in the spurs, similar to what we observe in M51.

\section{CONCLUSIONS}

We present deep, high-resolution $\mathrm{CO}(1-0)$ observations of a single field in M51. We determine that the extinction features seen in optical images of M51 (Scoville et al. 2001) are associated with gaseous structures of mass of a few $10^{6} M_{\odot}$. The length of these features is found to be a few hundred parsecs, consistent with previous measures of these features in extinction (La Vigne et al. 2006; Elmegreen 1980). Given this separation, the association with $\mathrm{H} \alpha$ emission, and a decoupling of the spur tip from the spiral arm pattern, we find that star formation in these spurs can result in previously unexplained interarm $\mathrm{H}$ II regions. The origin of these spurs and their importance to the overall population of interarm $\mathrm{H}$ II regions requires a wide-field survey of M51 and possibly other galaxies.

S. C. is supported by an NSF Graduate Research Fellowship. The Owens Valley Radio Observatory was funded in part by NSF grant AST 99-81546.
Briggs, D. S. 1995, BAAS, 187, 112.02

Brunner, G., et al. 2008, ApJ, 675, 316

Dame, T. M., Elmegreen, B. G., Cohen, R. S., \& Thaddeus, P. 1986, ApJ, 305, 892

Dobbs, C. L., \& Bonnell, I. A. 2006, MNRAS, 367, 873

Elmegreen, D. M. 1979, Ph.D. thesis, Harvard Univ. . 1980, ApJ, 242, 528

Kim, W. T., \& Ostriker, E. C. 2002, ApJ, 570, 132 2006, ApJ, 646, 213

La Vigne, M. A., Vogel, S. N., \& Ostriker, E. C. 2006, ApJ, 650, 818

Regan, M. W., Thornley, M. D., Helfer, T. T., Sheth, K., Wong, T., Vogel, S. N., Blitz, L., \& Bock, D. C. J. 2001, ApJ, 561, 218

Sanders, D. B., Scoville, N. Z., \& Solomon, P. M. 1985, ApJ, 289, 373

Sault, R. J., Teuben, P. J., \& Wright, M. C. H. 1995, in ASP Conf. Ser. 77, Astronomical Data Analysis Software and Systems IV, ed. R. A. Shaw, H. E. Payne, \& J. J. E. Hayes (San Francisco: ASP), 433

\section{REFERENCES}

Scoville, N. Z., Carlstrom, J. E., Chandler, C. J., Phillips, J. A., Scott, S. L., Tilanus, R. P. J., \& Wang, Z. 1993, PASP, 105, 1482

Scoville, N. Z., Polletta, M., Ewald, S., Stolovy, S. R., Thompson, R., \& Rieke, M. 2001, AJ, 122, 3017

Scoville, N. Z., \& Solomon, P. M. 1975, ApJ, 199, L105

Sheth, K., Vogel, S. N., Regan, M. W., Teuben, P. J., Harris, A. I., \& Thornley, M. D. 2002, AJ, 124, 2581

Sheth, K., Vogel, S. N., Wilson, C. D., \& Dame, T. M. 2008, ApJ, 675, 330 Sheth, K. S., Regan, M. W., Vogel, S. N., \& Teuben, P. J. 2000, ApJ, 532, 221 Shetty, R., \& Ostriker, E. C. 2006, ApJ, 647, 997

Vogel, S. N., Kulkarni, S. R., \& Scoville, N. Z. 1988, Nature, 334, 402

Wada, K. 2008, ApJ, 675, 188

Wada, K., \& Koda, J. 2004, MNRAS, 349, 270

Weaver, H. F. 1970, in IAU Symp. 39, Interstellar Gas Dynamics, ed. H. Habing (Dordrecht: Reidel), 22 